\title{
rnrfa: An R package to Retrieve, Filter and Visualize Data from the UK National River Flow Archive
}

by Claudia Vitolo, Matthew Fry and Wouter Buytaert

\begin{abstract}
The UK National River Flow Archive (NRFA) stores several types of hydrological data and metadata: daily river flow and catchment rainfall time series, gauging station and catchment information. Data are served through the NRFA web services via experimental RESTful APIs. Obtaining NRFA data can be unwieldy due to complexities in handling HTTP GET requests and parsing responses in JSON and XML formats. The rnrfa package provides a set of functions to programmatically access, filter, and visualize NRFA data using simple R syntax. This paper describes the structure of the rnrfa package, including examples using the main functions $\operatorname{gdf}()$ and $\operatorname{cmr}()$ for flow and rainfall data, respectively. Visualization examples are also provided with a shiny web application and functions provided in the package. Although this package is regional specific, the general framework and structure could be applied to similar databases.
\end{abstract}

\section{Introduction}

The increasing volume of environmental data available online poses non-trivial challenges for efficient storage, access and share of this information (Vitolo et al., 2015). An integrated and consistent use of data is achieved by extracting data directly from web services and processing them on-the-fly. This improves the flexibility of modelling applications allowing a more seamless workflow integration, and also avoids the need to store local copies that would need to be periodically updated, therefore reducing maintenance issues in the system.

In the hydrology domain, various data providers are adopting web services and Application Programming Interfaces (APIs) to allow users a fast and efficient access to public datasets, such as the National River Flow Archive (NRFA) hosted by the Centre for Ecology and Hydrology in the United Kingdom. The NRFA is a primary source of information for hydrologists, modellers, researchers and practitioners operating on UK catchments. It stores several types of hydrological data and metadata: gauged daily flow and catchment mean rainfall time series as well as gauging station and catchment information. Data are typically served through the NRFA web services via a web-based graphical user interface (http://nrfa.ceh.ac. uk/) and, more recently, via experimental RESTful APIs. REST (Representational State Transfer) is an architectural style that uses the HyperText Transfer Protocol (HTTP) to perform operations such as accessing resources on the web via a Uniform Resource Identifier (URI). In simple terms, the location of a NRFA dataset on the web is a unique string of characters that follows a pattern. This string is assembled using the rules described in the API documentation and can be tested by typing the string in the address bar of a web browser.

This paper describes the technical implementation of the rnrfa package (Vitolo, 2016). The rnrfa package takes the complexities related to web development and data transfer away from the user, providing a set of functions to programmatically access, filter, and visualize NRFA data using simple R syntax. Although the NRFA APIs are still in their infancy and prone to further consolidation and refinement, the experimental implementation of the rnrfa package can be used to test these data services and provide useful feedback to the provider.

The package is in line with a Virtual Observatory approach (Beven et al., 2012) as it can be used as back-end tool to link data and models in a seamless fashion. It complements R's growing functionality in environmental web technologies (Leeper et al., 2016), amongst which are rnoaa (Chamberlain, 2015, interface to NOAA climate data API), waterData (Ryberg and Vecchia, 2014, interface to the U.S. Geological Survey daily hydrologic data services) and RNCEP (Kemp et al., 2011, interface to NASA NCEP weather data).

This paper first presents the NRFA archive, its web services and related APIs. We then illustrate the design and implementation of the rnrfa package, and how it can be used in synergy with existing $R$ packages such as shiny (Chang et al., 2016), leaflet (Cheng and Xie, 2015), rmarkdown(Allaire et al., 2016), DT (Xie, 2015a), dplyr (Wickham and Francois, 2015) and parallel to generate interactive mapping applications, dynamic reports and big data analytics experiments. 


\section{NRFA web services}

The NRFA web services allow to view, filter and download data via a graphical user interface. This approach has a number of limitations. Firstly, time series of daily streamflow discharge and catchment rainfall can only be downloaded one at the time. Therefore, for large scale analyses, downloading datasets for hundreds of sites becomes a rather tedious task. Secondly, metadata can only be visualised (in table format) but not be downloaded. Metadata analyses may require copying and pasting large amounts of information introducing potential errors. Due to the above limitations, the NRFA is also accessible programmatically via a set of RESTful APIs. The API documentation is not in the public domain yet, therefore it must be considered experimental and subject to changes.

Station metadata (called catalogue hereafter) is available in JavaScript Object Notation (JSON) format. The catalogue contains a total of 18 attributes, which are listed in Table 1. The NRFA also provides time series of Gauged Daily Flow $\left(\mathrm{g} d f\right.$, in $\mathrm{m}^{3} / \mathrm{s}$ ) and Catchment Mean Rainfall (cmr, in mm per month), formatted in an XML variant called WaterML2 (http: //www . opengeospatial.org/ standards/waterml). WaterML2 is an Open Geospatial Consortium (OGC) standard used worldwide to rigorously and unambiguously describe hydrological time series. It builds upon existing standards such as Observations \& Measurements (Cox et al., 2011) for the metadata section and GML (Open Geospatial Consortium, 2013) for the observed time series. It is typically defined as a "Collection" and made up of five sections:

- The metadata section contains the document metadata (e.g., the generation date, the version, the generation system and the list of profiles utilised).

- The temporalExtent contains a description of the time period for which there are recordings (with a time stamp of start and end date).

- The localDictionary is a gml-based dictionary which stores the identifier (e.g., United Kingdom National River Flow Archive) and two dictionary entries: The first one describes the type of measurement (e.g., Gauged Daily Flow) with details on the variable measured (e.g., flow), units (e.g., $\mathrm{m} 3 / \mathrm{s}$ ) and frequency of measurements (e.g., daily); the second entry describes the gauging site with details on ratings and their limitations.

- The samplingFeatureMember describes the monitoring point (e.g., vertical datum and time zone) and the station owner.

- The observationMember contains a set of nodes which schema is borrowed from the OGC Observation and Measurement standard. This section contains a gml-based identifier (station identification number) and additional information, such as ObservationMetadata (contact info, identification info, etc.), phenomenonTime (beginning and end of recordings), ObservationProcess (process type and reference). Finally the sub-section result contains the measurements in a gml-based format.

The nested structure of the WaterML2 files makes parsing of long time series and related metadata relatively slow and complex. In order to improve access to NRFA's public data and metadata, we implemented a set of functions to assemble HTTP GET requests and parse XML/JSON responses from/to the catalogue and WaterML2 services using simple R syntax.

\section{Package availability and dependencies}

The rnrfa package is a package designed to extend basic $\mathrm{R}$ functionalities to interact with the NRFA. It builds on the following packages that should be installed beforehand: cowplot (Wilke, 2016), plyr (Wickham, 2011), httr (Wickham, 2016a), xml2 (Wickham and Hester, 2016), stringr (Wickham, 2016b), xts (Ryan and Ulrich, 2014), rjson (Couture-Beil, 2014), ggmap (Kahle and Wickham, 2013), ggplot2 (Wickham, 2009), rgdal (Bivand et al., 2016), sp (Pebesma and Bivand, 2005; Bivand et al., 2013) and parallel ${ }^{1}$. The stable version of the package is available on the Comprehensive R Archive Network repository (CRAN; https://CRAN. R-project. org/package=rnrfa/) and can be downloaded and installed by typing the following command in the R console:

> install.packages("rnrfa")

The development version is available from a GitHub repository (https://gi thub. com/cvitolo/rnrfa) and can be installed via devtools (Wickham and Chang, 2016), using the following commands:

$>$ install.packages ("devtools")

$>$ devtools: :install_github("cvitolo/rnrfa")

\footnotetext{
${ }^{1}$ In order to run the examples in this manuscript, the following packages should also be installed: shiny, leaflet, DT, ggrepel (Slowikowski, 2016), knitr (Xie, 2016, 2015b, 2014) and rmarkdown.
} 


\begin{tabular}{|c|c|c|}
\hline $\begin{array}{l}\text { Colum } \\
\text { number }\end{array}$ & $\begin{array}{l}\text { Column } \\
\text { name }\end{array}$ & Description \\
\hline 1 & id & Station identification number. \\
\hline 2 & name & Name of the station. \\
\hline 3 & location & Area in which the station is located. \\
\hline 4 & river & Name of the river catchment. \\
\hline 5 & stationDescription & $\begin{array}{l}\text { General station description, containing information on } \\
\text { weirs, ratings, etc. }\end{array}$ \\
\hline 6 & catchmentDescription & Information on topography, geology, land cover, etc. \\
\hline 7 & hydrometricArea & $\begin{array}{l}\text { UK hydrometric area identification number, the related } \\
\text { map is based on the Surface Water Survey designed } \\
\text { in the 1930s and available at http://www. ceh.ac.uk/ } \\
\text { data/hrfa/hydrometry/has.html. }\end{array}$ \\
\hline 8 & operator & $\begin{array}{l}\text { UK measuring authorities, the related map is available } \\
\text { at http://www. ceh.ac.uk/data/nrfa/hydrometry/ } \\
\text { mas.html. }\end{array}$ \\
\hline 9 & haName & Name of the hydrometric area. \\
\hline 10 & gridReference & The Ordnance Survey grid reference number. \\
\hline 11 & stationType & Type of station (e.g., flume, weir, etc.). \\
\hline 12 & catchmentArea & Catchment area in $\mathrm{Km}^{2}$. \\
\hline 13 & gdfStart & First year of monitoring. \\
\hline 14 & gdfEnd & Last year of monitoring. \\
\hline 15 & farText & Information on the regime (e.g., natural, regulated, etc.). \\
\hline 16 & categories & $\begin{array}{l}\text { Various tags (e.g., FEH_POOLING, FEH_QMED, HI- } \\
\text { FLOWS_INCLUDED). }\end{array}$ \\
\hline 17 & altitude & $\begin{array}{l}\text { Altitude measured in metres above Ordnance Datum or, } \\
\text { in Northern Ireland, Malin Head. }\end{array}$ \\
\hline 18 & sensitivity & $\begin{array}{l}\text { Sensitivity index calculated as the percentage change in } \\
\text { flow associated with a } 10 \mathrm{~mm} \text { increase in stage at the } \\
Q_{95} \text { flow. }\end{array}$ \\
\hline
\end{tabular}

Table 1: Gauging station metadata, more detail is provided at http://www.ceh.ac.uk/data/nrfa/ data/gauging_stations.html.

The package is loaded using the following command:

$>$ library (rnrfa)

The package is fully documented and additional sample applications are available on the dedicated web page http://cvitolo. gi thub.io/rnrfa/. Feedbacks and contributions can be submitted through the GitHub issue tracking system (https://github.com/cvitolo/rnrfa/issues) and pull requests (https://github.com/cvitolo/rnrfa/pulls), respectively.

\section{Design and implementation}

In many hydrological analyses the importance of efficient data retrieval is often underestimated with the consequence of allocating more time to this first task then to the data processing and analysis of results. The rnrfa packages provides re-usable functions, based on a consistent syntax, that attempts to simplify data retrieval and makes it scalable to multiple data requests.

\section{Catalogue metadata}

The full list of gauging stations is in JSON format and can be retrieved using the function catalogue(), used with no inputs.

$>$ allstations <- catalogue()

This converts the information into a data frame with one row per station and 18 columns (Table 1 contains a detailed description of the attributes). The reader should note that the server response includes the Ordnance Survey (OS) grid reference, not latitude and longitude coordinates. The 
catalogue() function converts the grid reference to latitude and longitude, then joins the coordinates to the data frame containing the list of stations.

The conversion is handled by the osg_parse() function which can transform OS grid references of different lengths to: a) latitude and longitude, in the WSGS $84^{2}$ coordinate system; b) easting and northing, in the $\mathrm{BNG}^{3}$ coordinate system. This function accepts two arguments: gridRef, a character string containing the OS grid reference, and CoordSystem, that can be either "WGS84" (default) or "BNG". The code below shows how to convert an example OS grid reference, "NC581062", to the two types of coordinates.

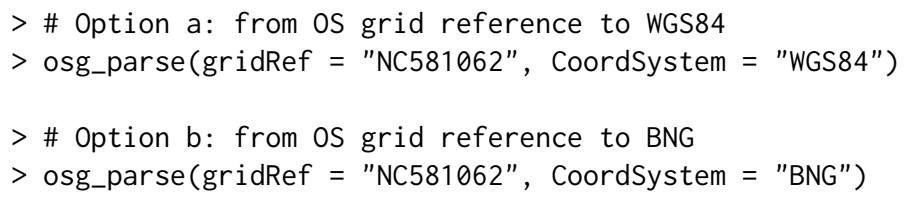

\section{Filtering stations}

The catalogue() function provides 5 optional arguments that can be used to filter metadata based on various criteria. The argument all, for instance, is TRUE by default and forces all the metadata to be retrieved. If all is set to FALSE, the resulting data frame contains only the following columns: id, name, river, catchmentArea, lat, lon. This can be used, for instance, to print a concise version of the table to the screen.

At the time of writing, 1539 stations are monitored within NRFA. Very rarely the full set of stations is used. Depending on the aim of the analysis, stations might need to be filtered based on a geographical bounding box, length of the recording period, thresholds, etc. Below are some examples showing how to filter stations based on one or multiple criteria.

Filtering based on a geographical bounding box. Stations can be filtered based on a bounding box thanks to the NRFA web service and a specific functionality of its API. A bounding box should be defined as a list of four named elements (minimum longitude, minimum latitude, maximum longitude and maximum latitude) and passed as input to the catalogue() function using the argument bbox. The following example shows how to define a bounding box for the Plynlimon area (mid-Wales, United Kingdom), filter the related stations and map their location using the ggmap package. In Figure 1 the location of each station is shown as a red dot, while the name of the station is used as a label.

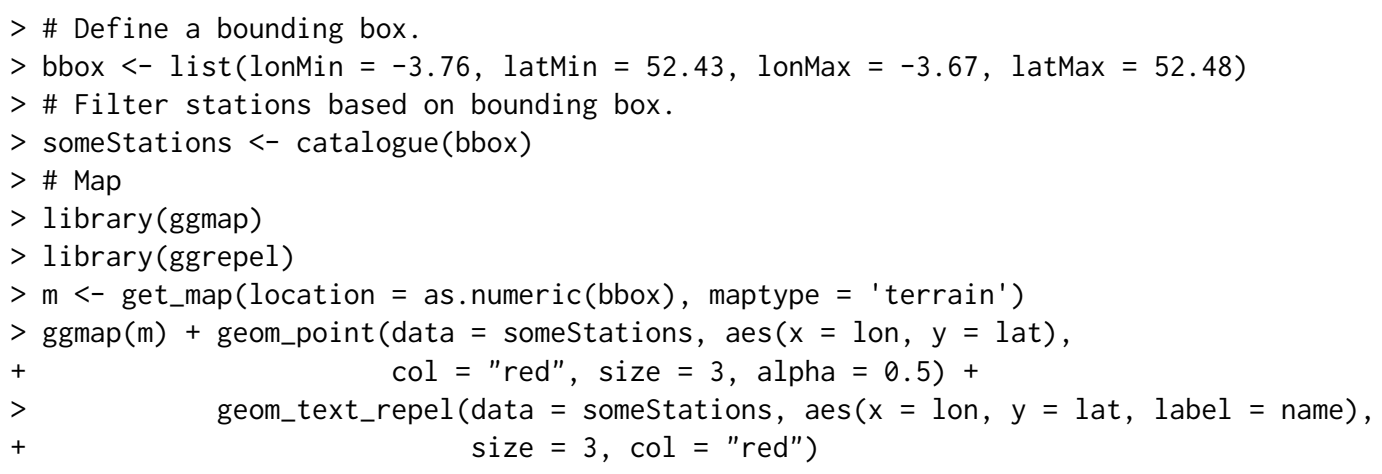

Filtering based on recording period. To calculate summary statistics, it is often useful to select only stations with at least $x$ number of recording years. In the example below, we select only gauging stations with a minimum of 100 years of recordings, using the argument minRec. The result is a list of three stations, two of which are located in South England and one in Wales.

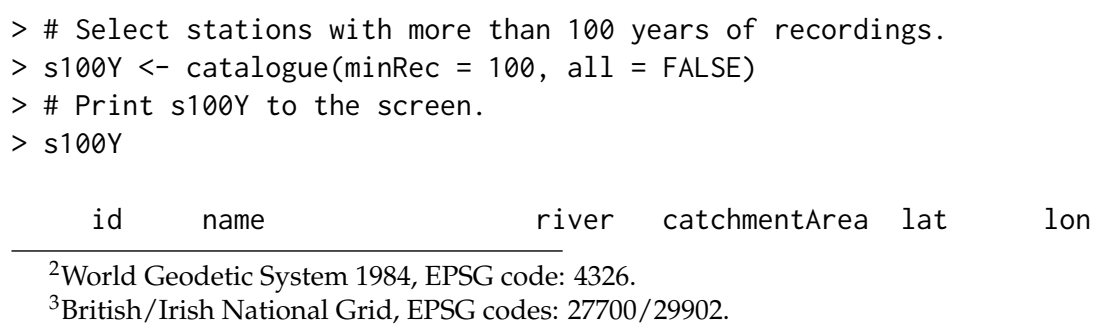




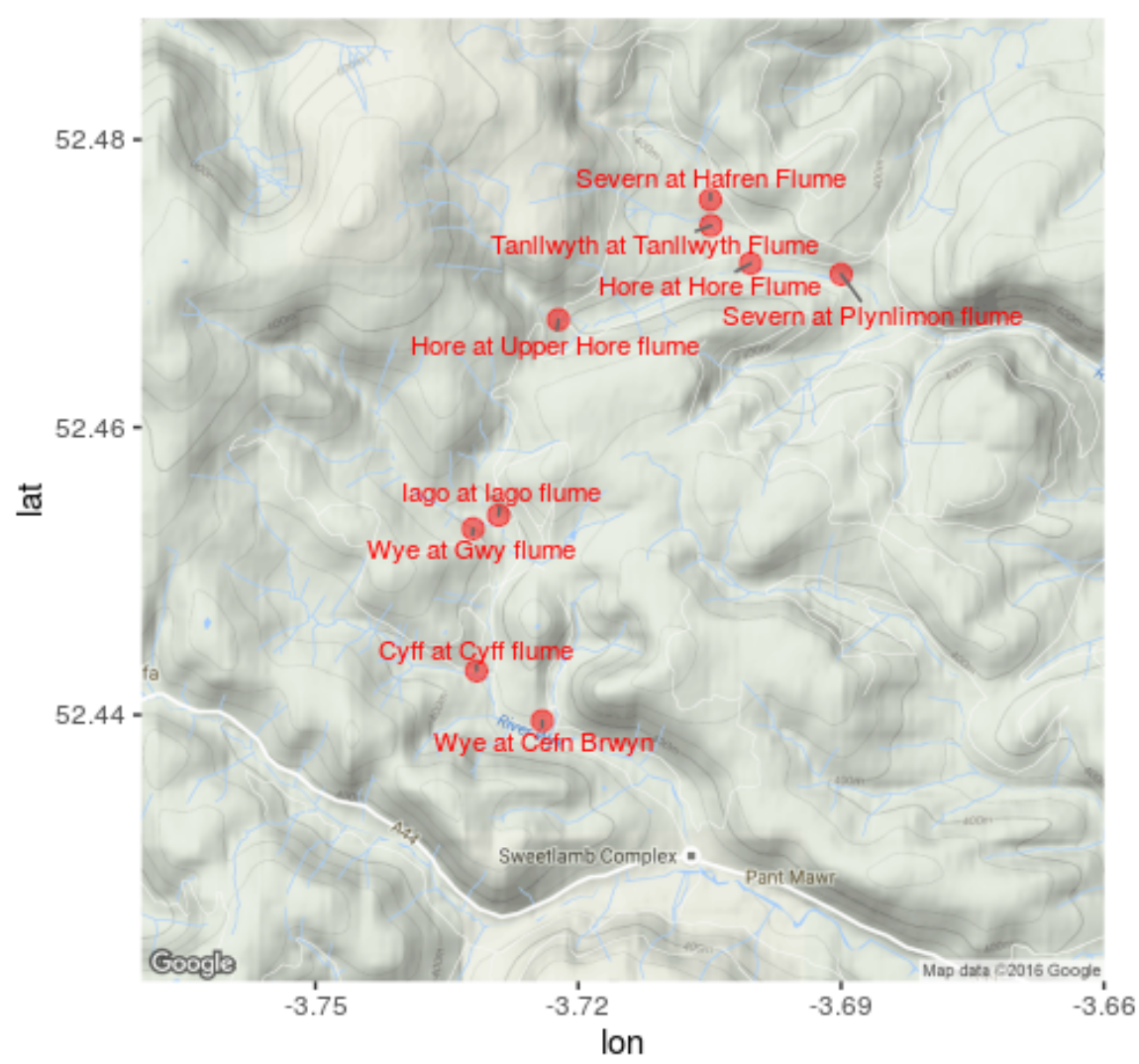

Figure 1: Map of Plynlimon area with NRFA selected gauging stations (red dots).

$\begin{array}{llllllr}636 & 38001 & \text { Lee at Feildes Weir } & \text { Lee } & 1036 & 51.76334 & 0.01277874 \\ 665 & 39001 & \text { Thames at Kingston } & \text { Thames } & 9948 & 51.41501 & -0.30887638 \\ 1130 & 55032 & \text { Elan at Caban Dam } & \text { Elan } & 184 & 52.26907 & -3.57239164\end{array}$

Filtering based on metadata entries. It is also possible to filter stations based on a number of metadata entries using the arguments: columnName (name of the column to filter) and columnvalue (string or numeric value to match or compare). The function catalogue() looks for records containing the string columnValue in the column columnName. If columnName refers to a character field, the search is case sensitive and can be used to filter the stations based on the river name, catchment name, location and so on. In the example below we filter 34 stations falling within the Wye (Hereford) hydrometric area:

$>$ stationsWye $<-$ catalogue $($ columnName $=$ "haName", columnValue = "Wye (Hereford)" $)$

If columnName refers to a numeric field and columnValue contains special characters such as $>,<, \geq$ and $\leq$ followed by a number, stations are filtered using a threshold. For instance, there are 7 stations with drainage area smaller than $1 \mathrm{Km}^{2}$, which can be filtered using the command below:

$>$ stations1KM <- catalogue $($ columnName $=$ "catchmentArea", columnValue $="<1 "$ )

\section{Combined filtering}

Filtering capabilities can also be combined. In the example below we filter all the stations within the above defined bounding box that belong to the Wye (Hereford) hydrometric area and have a minimum of 50 years of recordings. The only station that satisfies all the criteria is the Wye at Cefn Brwyn.

$>$ catalogue (bbox, columnName = "haName", columnValue = "Wye (Hereford)",

$+\quad \operatorname{minRec}=50$, all $=$ FALSE $)$

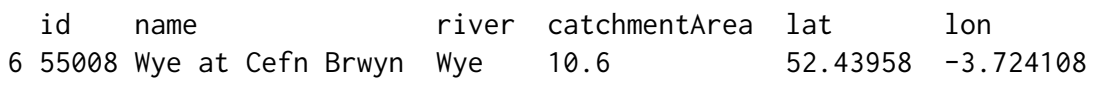




\section{WaterML2 services}

Once a certain number of stations are selected, time series of gauged daily flow and catchment mean rainfall data can be obtained by requesting access to the NRFA WaterML2 service using the functions $\operatorname{gdf}()$ and $\operatorname{cmr}()$, respectively. These functions assemble and send data requests to the WaterML2 service, parse responses and convert them to a time series object (of class from package xts). They use the same syntax and require the following arguments:

- id, the station identification numbers. This can either be a single string or a character vector.

- metadata, a logical variable. If set to FALSE (default), metadata are not parsed. If it is set to TRUE, the result for a single station is a list of two named elements: data (time series) and meta (metadata).

When $\operatorname{gdf}()$ and $\operatorname{cmr}()$ are executed, the assembled data request is printed to the screen. This is very useful if the user wants to understand how the API works behind the scenes, but not when incorporating the code in automated scripts. Although the NRFA API documentation is not public yet, the patterns are simple and can be easily extrapolated running a few examples.

\section{Get gauged daily flow}

Raw flow data are typically measured in $\mathrm{m}^{3} / \mathrm{s}$, at 15-minute intervals. Data are first quality controlled, then the daily mean is calculated and stored in the NRFA public database. These data are typically collected for the monitoring of river networks but can also be used to calibrate hydrological models and build forecasting systems. The example below shows how to get the daily flow for the Tanllwyth at Tanllwyth Flume and the assembled data request (printed to the console).

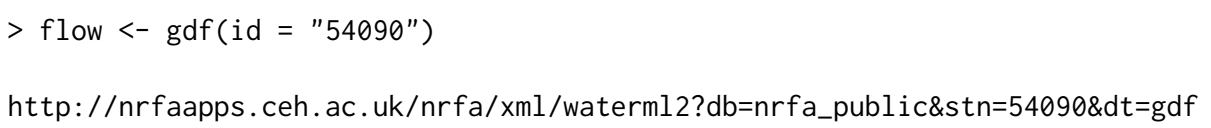

The result is a time series (of class "xts"). No station-specific information is stored, because the argument metadata is set to FALSE by default. An "xts" object can be easily converted into a data frame object and exported to a text file (e.g., csv) for use in other modelling software, as demonstrated in the example below.

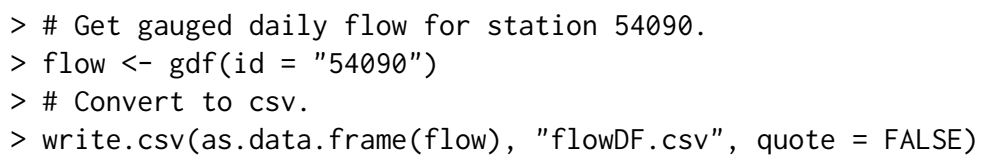

\section{Get catchment mean rainfall}

The main forcing input in any hydrological model is rainfall. In many cases it is important to calculate the average rainfall over a catchment, this is achieved by using geospatial interpolation methods or, more simplistically, calculating the weighted average using a number of weather stations within the catchment and/or in the nearby areas. The NRFA provides pre-calculated monthly catchment mean rainfall, measured in $\mathrm{mm}$, for a number of UK catchments. As the calculation is consistent across catchments, these datasets are a valuable resource to ensure reproducibility of hydrological analyses. Similar to $\operatorname{gdf}()$, the function $\mathrm{cmr}()$ allows users to retrieve the catchment mean rainfall data by specifying the argument $i d$. The example below shows that, if we set the argument metadata to TRUE, we can use metadata to automatically populate title and labels in a plot, as in Figure 2. The reader should note that rain $\$$ data is an "xts" object, therefore plot (rain\$data) uses the S3 method for "xts".

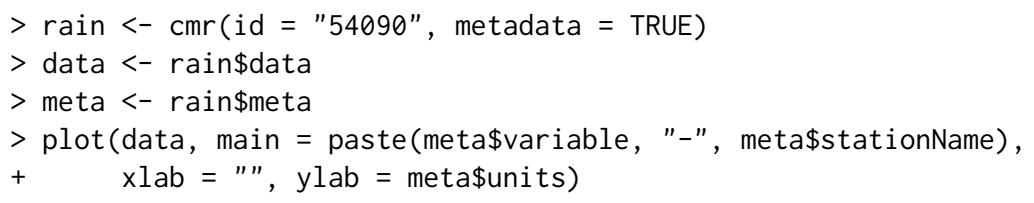

Station information consists of: the station name, location in latitude and longitude coordinates, the variable measured (i.e., rainfall), units (i.e., $\mathrm{mm}$ ), aggregation function (i.e., accumulation), time step of recording (i.e., month) and time zone. 


\section{Rainfall - Tanllwyth at Tanllwyth Flume}

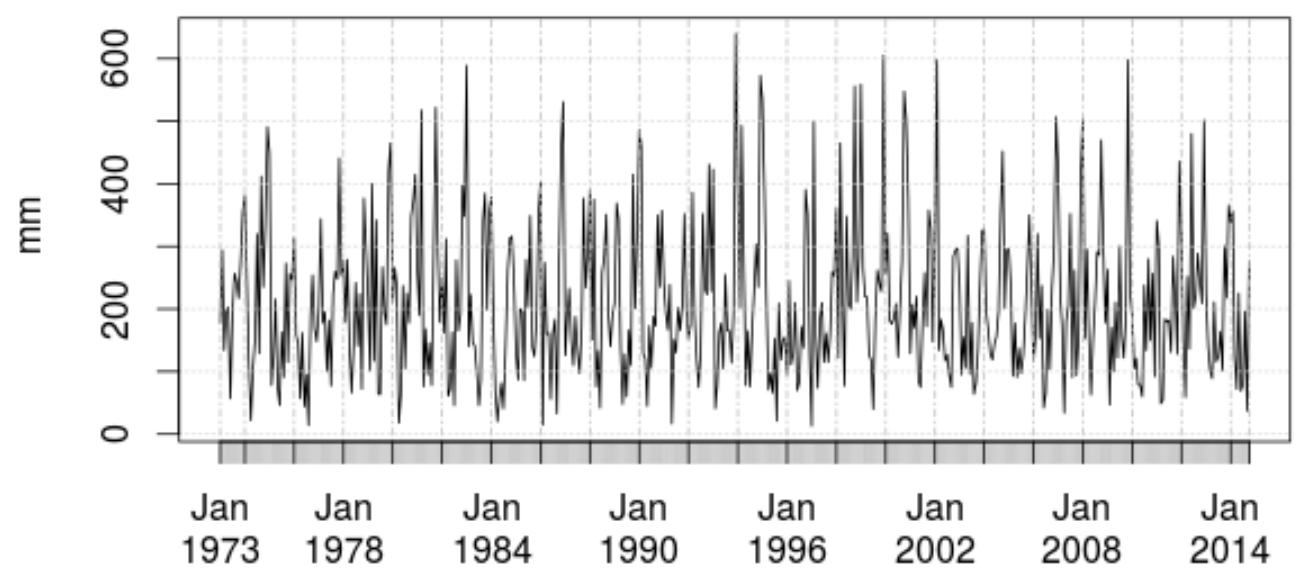

Figure 2: Monthly catchment mean rainfall for the Tanllwyth at Tanllwyth Flume catchment.

\section{Tanllwyth at Tanllwyth Flume}

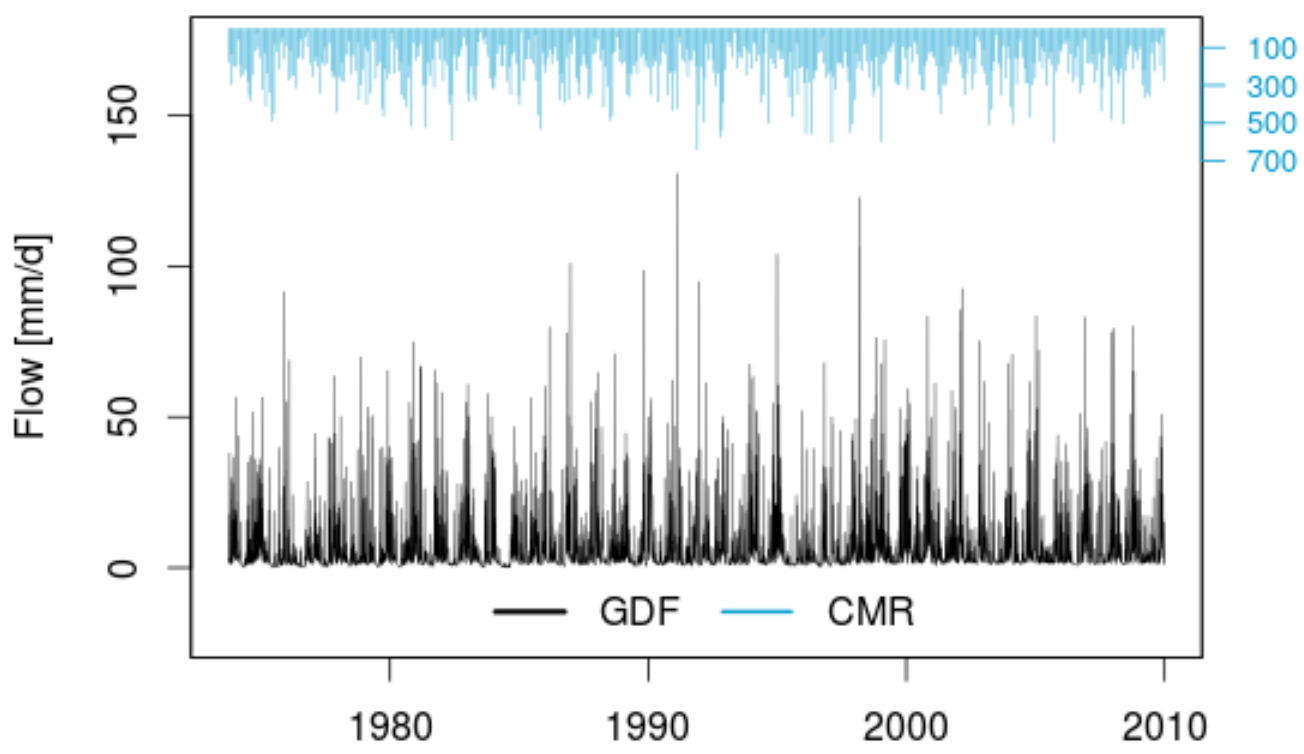

Figure 3: Monthly catchment mean rainfall and daily flow for the Tanllwyth at Tanllwyth Flume catchment.

\section{Convert and compare flow and rainfall for a given catchment}

In the NRFA, flow and rainfall are stored in $\mathrm{m}^{3} / \mathrm{s}$ and $\mathrm{mm} / \mathrm{month}$, respectively, therefore they are not directly comparable. However, given the catchment area (from the metadata catalogue), the flow can be easily converted into $\mathrm{mm}$ / day and then compared to the rainfall, for instance by plotting them on the same time line. Although the operations are trivial, it is a relatively lengthy procedure that can be simplified using the function plot_rain_flow(). This function uses the station id as input to request metadata as well as flow and rainfall time series for the given catchment, converts the flow from its original units to $\mathrm{mm} /$ day and then plots the converted flow and rainfall on two different $y$-axes so that they can be visually compared, as shown in Figure 3.

$>$ plot_rain_flow(id $=$ "54090")

\section{Multiple sites}

The package rnrfa is particularly useful for large scale data acquisition. If the id argument is a vector, the functions $\mathrm{gdf}()$ and $\mathrm{cmr}()$ can be used to sequentially fetch time series (meta)data from multiple 


\begin{tabular}{crrrrrrr}
\hline Test & \multicolumn{1}{c}{$\min$} & \multicolumn{1}{c}{ lq } & \multicolumn{1}{c}{ mean } & \multicolumn{1}{c}{ median } & \multicolumn{1}{c}{ uq } & max & neval \\
\hline $\mathrm{a}$ & 17.598647 & 17.95601 & 18.419300 & 18.355630 & 19.037328 & 19.16267 & 10 \\
$\mathrm{~b}$ & 3.564888 & 8.91512 & 8.411546 & 9.504491 & 9.666812 & 10.58291 & 10 \\
\hline
\end{tabular}

Table 2: Benchmark tests comparing retrieval time for sequential (a) and simultaneous calls to the server (b). Results show time in seconds, obtained by averaging over 10 repetitions using the microbenchmark package (Mersmann, 2015).

sites. As the server can handle multiple requests, concurrent calls can be sent simultaneously using the parallel package. In order to send concurrent calls, a cluster object, created by the parallel package, should be passed to $\operatorname{gdf}()$ or $\mathrm{cmr}()$ using the argument $\mathrm{cl}$. Below is a simple benchmark test in which we compare the processing time for collating flow time series data for the 9 stations in the Plynlimon area sending: a) 1 data request at the time and b) 9 simultaneous requests. The operations are repeated 10 times. The results are averaged and summarised in Table 2, which shows that (a) takes about 18 seconds, while (b) about 8 seconds. The reader should note that the time for retrieval does not reduce proportionally with the number of simultaneous requests because there is a limit in the number of calls the server can handle, which depends on the infrastructure and the number of incoming requests from other users.

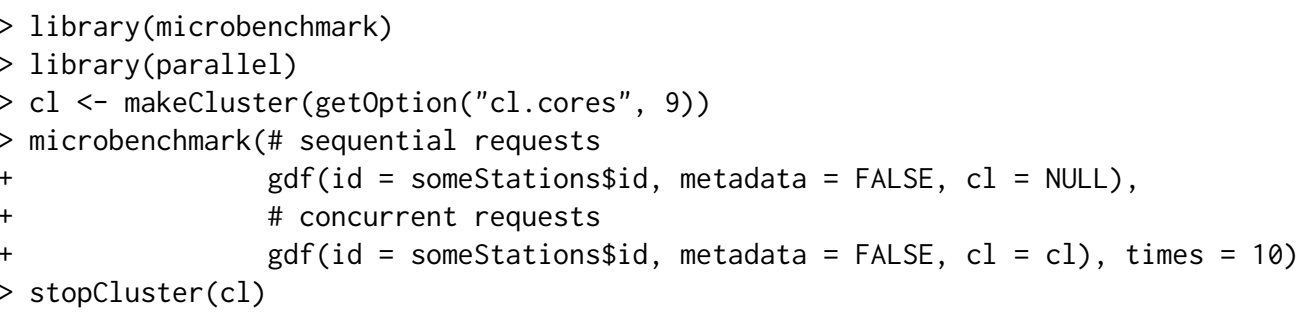

\section{Some applications}

The rnrfa package is an ideal building block for many scientific workflows but can also work as back-end tool for a number of web applications, from interactive mapping and dynamic reports that improve reproducibility of analysis, to the integration into more sophisticated big data analytics experiments. This can be achieved thanks to the intrinsic interoperability of the R environment. Some example applications are given in the following sections.

\section{Dynamic mapping and reporting application}

Here we demonstrate the generation of a dynamic mapping and reporting application to summarise stations' metadata and map the spatial distribution of the monitoring network for each operator. The user can select the name of the operator using a drop-down menu and the dynamic document automatically renders an interactive map showing a marker for each station in the network on top of a background map based on OpenStreetMap. Users can zoom in/out and navigate to a specific area. Finally, the user can click on a marker to read name and station identification number from a pop-up window. Figure 4 shows a screenshot of the web application. At the bottom of the page is a dynamic table that summarises the metadata associated with the selected stations in the network. The table can be filtered using an interactive search box. The textual content also updates automatically the reporting of the number of stations within the selected network. The web application depends on the following packages: rmarkdown, knitr, shiny, leaflet and DT and its source code is available as gist at the following URL: https://gist.github.com/cvitolo/d5d46b5e8f3676013857.

\section{Geoprocessing based on user-defined areas}

The NRFA web site does not allow users to execute geoprocessing tasks, for instance, to intersect the list of stations with user-defined or externally sourced areas. In some cases it might be of interest to explore the distribution of stations based on high-level administrative boundaries such as regions/countries. This is useful to understand whether there are differences in the reliability of the networks that can be explained by the different management approaches. Eurostat established a hierarchy of three levels of administrative divisions within each European country, called Nomenclature of Territorial Units for 


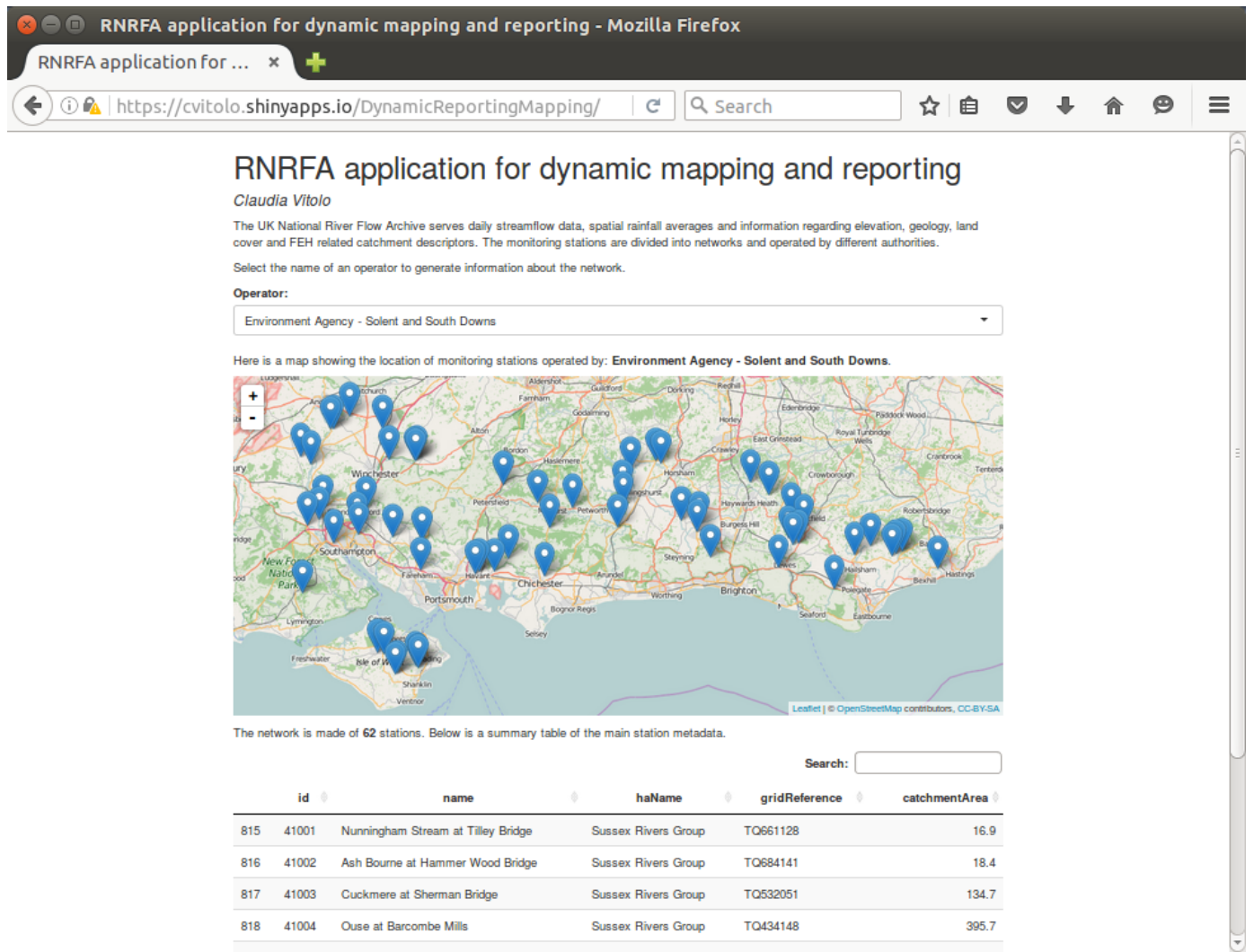

Figure 4: RNRFA application for dynamic reporting and mapping.

Statistics (NUTS) ${ }^{4}$. At the first level, UK is divided into 12 regions: Northern Ireland, Scotland, Wales and 9 English sub-regions (East Midlands, East of England, Greater London, North East, North West, South East, South West, West Midlands, Yorkshire and the Humber). Calculating, for instance, the number/density of stations by region is not possible using the NRFA web site because the stations' metadata does not contain information on this type of administrative region and users cannot specify their own. However, these simple geoprocessing operations become relatively trivial using the rnrfa package.

The procedure consists of five steps:

- retrieve the list of NRFA stations (using the catalogue() function);

- load the NUTS (level 1) shapefile and reproject the polygon to the geographic coordinate system WGS84 (using the rgdal and sp packages);

- transform the NRFA list of stations into a SpatialPointDataFrame ;

- spatially overlay NRFA stations (points) and NUTS1 regions (polygons);

- add a new column, containing the name of the NUTS1 regions, to the list of NRFA stations.

The updated list of stations is included, as sample dataset, in the data folder of this package, under the name stationSummary. Table 3 summarises the number of stations per region, the area of each region (in $\mathrm{Km}^{2}$ ), and the density of stations (number of stations $/ \mathrm{Km}^{2}$ ). The metadata can now be easily summarised by NUTS1 region, for instance the boxplot in Figure 5 shows the distribution of years of recording. Northern Ireland seem to have the youngest network, with recording years in the range $[16,44]$. Only three regions have stations with more than 100 years of recordings: East of England, London and Wales. Scotland and Northern Ireland have the lowest density of gauging stations, while Greater London the highest. The code to reproduce this example is available as gist at the following URL: https://gist.github.com/cvitolo/aa3bc6f08a8394f653442e276568f9b3.

\footnotetext{
${ }^{4}$ The Nomenclature of Territorial Units for Statistics (NUTS), is a standard for referencing the administrative divisions of European countries. There are three levels of NUTS and a shapefile is available from the Eurostat web site (http://ec. europa. eu/eurostat/cache/GISCO/geodatafiles/NUTS_2013_01M_SH.zip).
} 


\begin{tabular}{rllrrr}
\hline & NUTS_ID & Region & \# stations & Area $\left(\mathrm{Km}^{2}\right)$ & Density \\
\hline 1 & UKC & North East (England) & 54.00 & 8601.77 & 0.006 \\
2 & UKD & North West (England) & 137.00 & 14170.34 & 0.010 \\
3 & UKE & Yorkshire and the Humber & 102.00 & 15418.70 & 0.007 \\
4 & UKF & East Midlands & 101.00 & 15637.21 & 0.006 \\
5 & UKG & West Midlands & 103.00 & 12999.97 & 0.008 \\
6 & UKH & East of England & 149.00 & 19159.91 & 0.008 \\
7 & UKI & Greater London & 36.00 & 1575.97 & 0.023 \\
8 & UKJ & South East (England) & 169.00 & 19105.67 & 0.009 \\
9 & UKK & South West (England) & 176.00 & 23912.24 & 0.007 \\
10 & UKL & Wales & 132.00 & 20817.37 & 0.006 \\
11 & UKM & Scotland & 324.00 & 78984.40 & 0.004 \\
12 & UKN & Northern Ireland & 56.00 & 14175.46 & 0.004 \\
\hline
\end{tabular}

Table 3: Summary of number of stations per NUTS1 region, area of each region and density of stations.

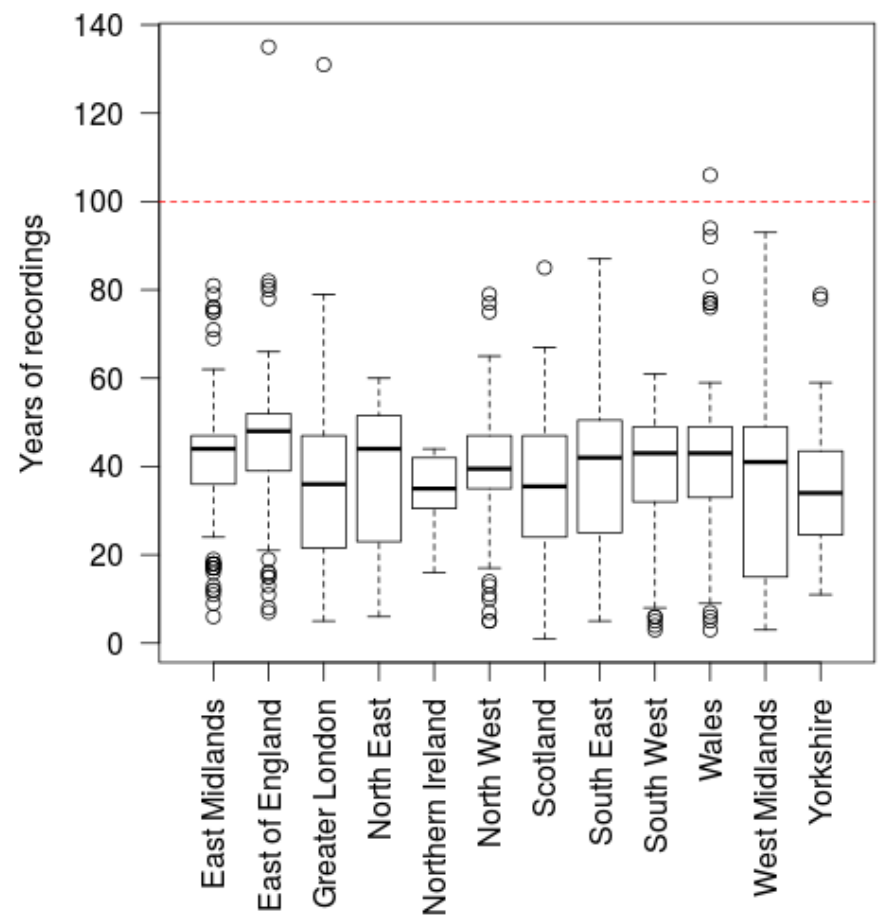

Figure 5: Distribution of recording years for NRFA stations by NUTS1 regions.

\section{Big data analytics experiment}

In the last few years, the UK MetOffice has reported "unusual warmth and lack of rainfall during March and April, particularly over England and Wales" ${ }^{5}$. Dry springs can affect water resources, because river flow below average translates, for instance, in reduced availability of drinking water. In this section we present a big data analytics experiment in which we try to understand if there is any evidence, in the NRFA data, that springs in the UK are becoming drier, both in terms of rainfall and river flow. This type of experiment consists of retrieving all the available rainfall and flow time series and find out, for each station, whether there is an increasing/decreasing trend.

Using the NRFA web site, the comparison of time series is only feasible for a limited number of sites. Time series should be first downloaded as text files and then compared manually. The biggest advantage of using the rnrfa package, instead, is that multiple downloads can be automated using a single line of code.

In this experiment we used a cluster of 64 cores to download and analyse all the time series available from the NRFA stations with more than 10 years of recordings. The time series were first

5 http://www.metoffice.gov.uk/climate/uk/interesting/2011_spring 
A

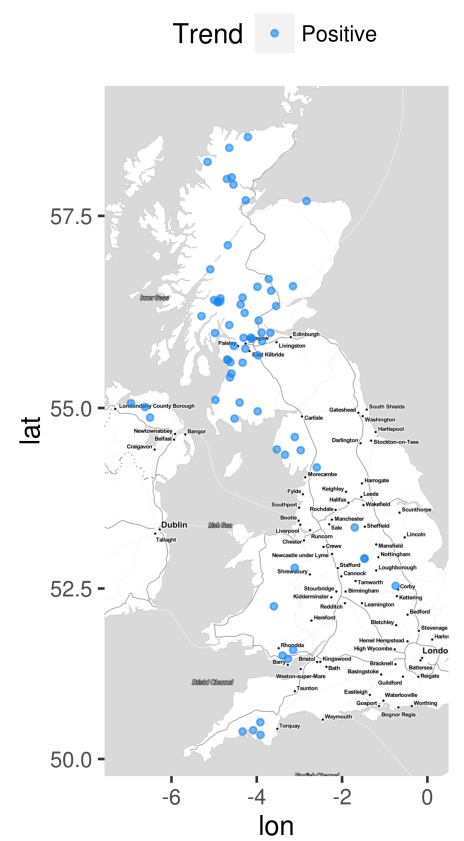

B

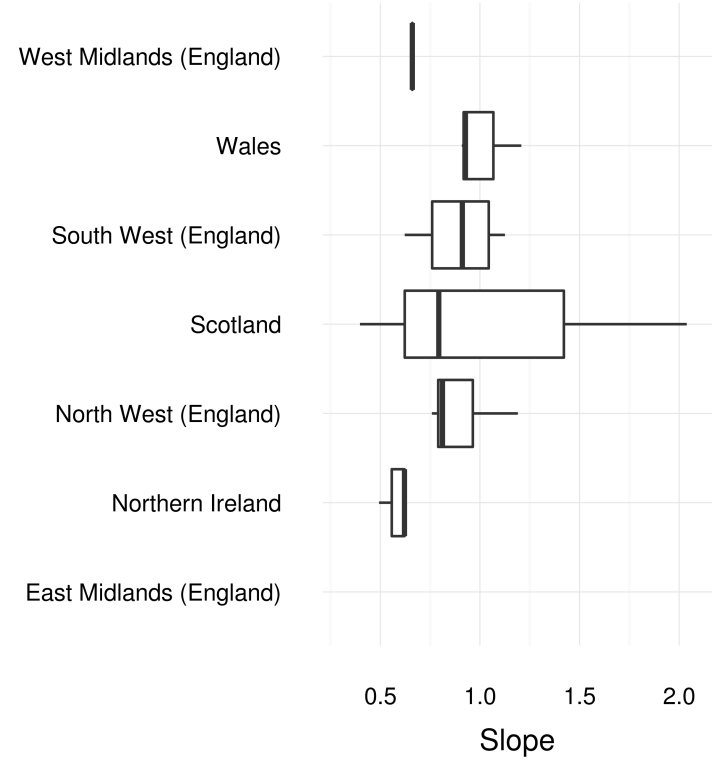

Figure 6: Map and boxplot of rainfall trend during spring.

downloaded, then summarised in terms of annual averages over the spring period. Seasonal averages can be calculated using the function seasonal_averages(), which takes as input a time series and a period of interest (e.g., spring) and calculates the related annual average. Using a very simplistic approach, a linear model was fit to the annual averages and the slope coefficient was used to estimate the trend. Negative slopes correspond to decreasing flow/rainfall, while positive slopes correspond to an increase of flow/rainfall over time. Once the fitted slope is calculated for each station, the results can be plotted using the function plot_trend(). Figures 6 and 7 show only the statistically significant trends for rainfall and flow respectively. Each figure is divided into two plots: Plot A shows the spatial distribution of negative trends with red dots and positive trends with blue dots; plot B shows the variability of trends over NUTS1 regions. In the latter plot, outliers are removed by showing only values between the 5th and 95th quantiles. From a meteorological perspective (Figure 6), there are only positive statistically significant trends and Scotland shows the largest. In terms of hydrological responses (Figure 7), trends are more subtle as the interquartile range is concentrated around zero. The most extreme negative trends were found in Scotland and North East England.

The entire run took about 31 minutes, the code to reproduce this example is available as gist at the following URL: https://gist.github. com/cvitolo/612eb2ae9b47fe8f11a1ed8d06e3b434. There are certainly more rigorous methodologies to estimate seasonal trends. This experiment was just an attempt to demonstrate that the rnrfa can simplify large scale data acquisition tasks.

\section{A note on package usage}

The cranlogs (Csardi, 2015) package provides an API interface to download logs from the RStudio CRAN mirror which contains download counts from unique IP addresses and can be used as a proxy to estimate the volume of package users. By September 2016, the rnrfa package had been downloaded from CRAN 6372 times, just from this mirror, following a trend very similar to the waterData package (see Figure 8). Because the RStudio mirror is located in the US, it is expected that the download counts from UK mirrors could be even higher. We derive that this package is of interest for a large community of users, which gives us scope for future developments.

\section{Summary}

This article describes the rnrfa package for interacting programmatically with the UK National River Flow Archive. It allows to access web resources such as the catalogue of stations' metadata and the WaterML2 service to retrieve gauged daily flow and (monthly) catchment mean rainfall. The package provides functions to query the catalogue based on various criteria (e.g., geographical bounding 
A

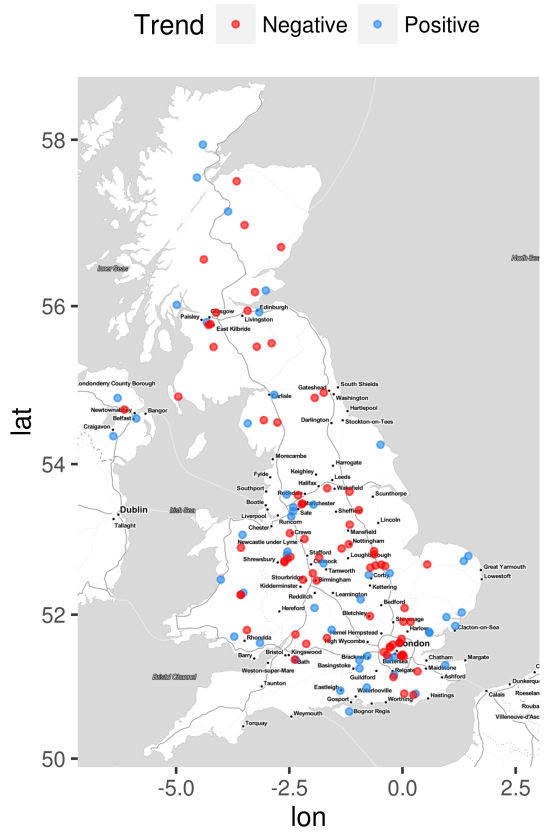

B

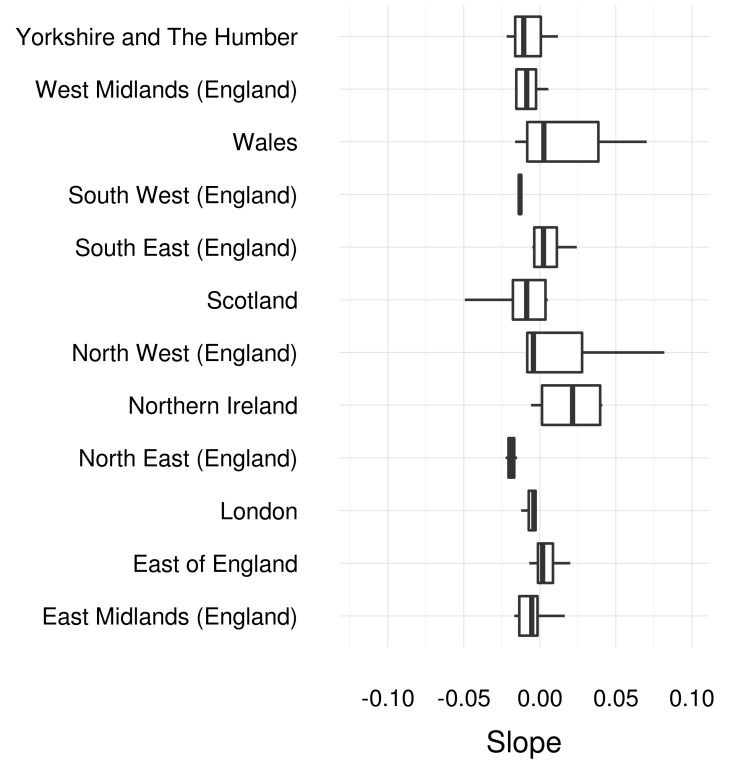

Figure 7: Map and boxplot of flow trend during spring.

Download counts from RStudio CRAN mirror

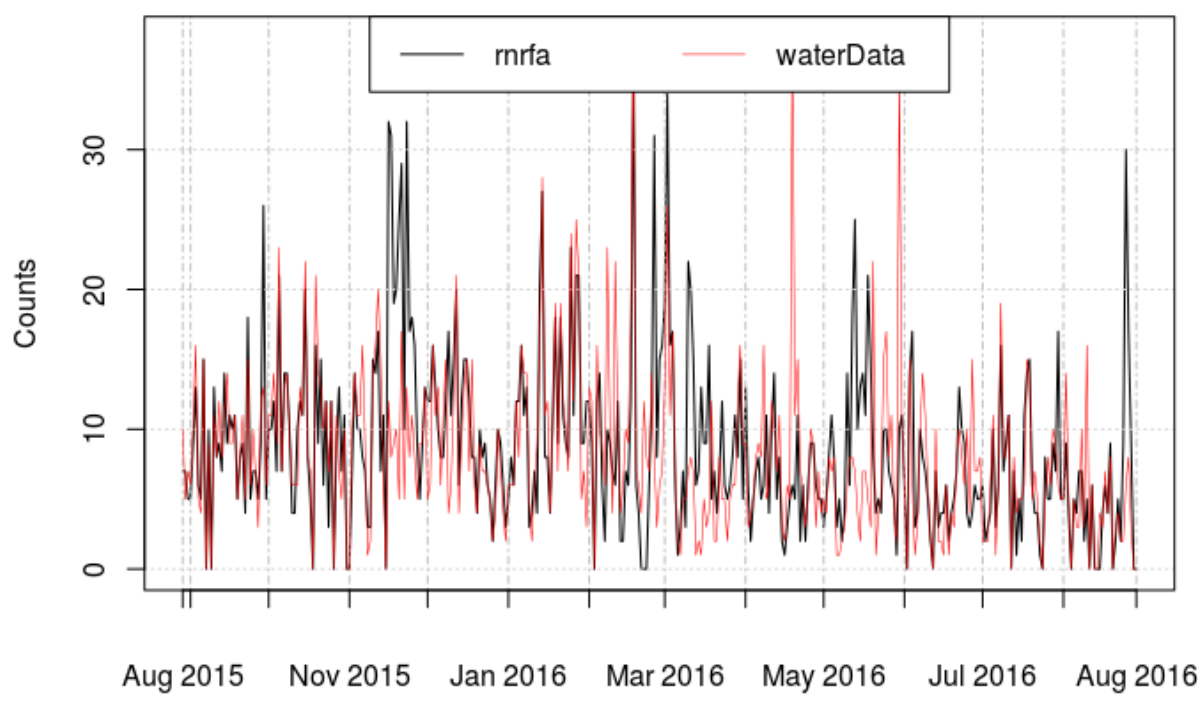

Figure 8: Comparison between rnrfa and waterData download counts from independent IP addresses.

box, minimum number of recording years, river catchment/hydrometric area/operators amongst many other options), retrieve and visualise flow and rainfall time series, convert coordinates and flow measurements, and plot basic seasonal trends grouped on user defined regions. Some of these capabilities are strongly linked to the particular content of the NRFA database and are not directly transferable/applicable to other data sources. However the $g d f()$ and $\operatorname{cmr}()$ functions could be re-used, with minimal changes, to get data/metadata from other providers adopting the WaterML2 standard.

The package is a convenient standalone application that allows NRFA users a more efficient access to the public database, compared to the web interface, e.g., the possibility to efficiently retrieve data from multiple sites. The rnrfa package can also be used as back-end tool for web applications. Amongst the existing R interfaces to data APIs, rnrfa follows a logic similar to waterData: Sites are first identified through a catalogue, streamflow data are imported via the station identification number, 
then data are visualised and/or used in analyses. However, our package does not implement any function for data cleanup, because NRFA data are highly quality controlled. Users can currently take advantage of other packages such as xts to calculate aggregate variables, evd (Stephenson, 2002) for the analysis of extreme events, outliers (Komsta, 2011) to identify possible outliers and sp and spacetime (Pebesma, 2012; Bivand et al., 2013) for more advanced spatio-temporal processing.

In the future, we plan to implement additional processing functions (e.g., to compare gdf with flow in bankfull condition which is highly important for flood frequency estimations). Further developments are also scheduled on the NRFA side to include Web Feature Service (WFS), Sensor Observation Services (SOS) and updates to WaterML2 OGC standards. WFS layers can already be loaded and manipulated using rgdal (Bivand et al., 2016), while sos4R (Nüst et al., 2011) can be used as client for SOS.

\section{Acknowledgments}

This work was carried out when Claudia Vitolo was working at the Imperial College London and supported by the Natural Environment Research Council pilot Probability, Uncertainty \& Risk in the Environment (PURE) NE/I004017/1. Comments from two referees are gratefully acknowledged.

\section{Bibliography}

J. Allaire, J. Cheng, Y. Xie, J. McPherson, W. Chang, J. Allen, H. Wickham, A. Atkins, and R. Hyndman. rmarkdown: Dynamic Documents for R, 2016. URL https://CRAN.R-project.org/package= rmarkdown. R package version 0.9.2. [p102]

K. Beven, W. Buytaert, and L. Smith. On virtual observatories and modelled realities (or why discharge must be treated as a virtual variable). Hydrological Processes, 26:1906-1909, 2012. doi: 10.1002/hyp. 9261. [p102]

R. Bivand, T. Keitt, and B. Rowlingson. rgdal: Bindings for the Geospatial Data Abstraction Library, 2016. URL https: //CRAN. R-project.org/package=rgdal. R package version 1.1-10. [p103, 114]

R. S. Bivand, E. Pebesma, and V. Gomez-Rubio. Applied Spatial Data Analysis with R. Springer-Verlag, 2nd edition, 2013. doi: 10.1007/978-1-4614-7618-4. [p103, 114]

S. Chamberlain. rnoaa: 'NOAA' Weather Data from R, 2015. URL https://CRAN.R-project.org/ package=rnoaa. R package version 0.5.0. [p102]

W. Chang, J. Cheng, J. Allaire, Y. Xie, and J. McPherson. shiny: Web Application Framework for R, 2016. URL https: //CRAN. R-project.org/package=shiny. R package version 0.13.2. [p102]

J. Cheng and Y. Xie. leaflet: Create Interactive Web Maps with the JavaScript "Leaflet" Library, 2015. URL https://CRAN.R-project.org/package=leaflet. R package version 1.0.0. [p102]

A. Couture-Beil. rjson: JSON for $R, 2014$. URL https://CRAN.R-project.org/package=rjson. R package version 0.2.15. [p103]

S. Cox et al. Observations and measurements - XML implementation. OGC document, 2011. [p103]

G. Csardi. cranlogs: Download Logs from the "RStudio" CRAN Mirror, 2015. URL https://CRAN. Rproject.org/package=cranlogs. R package version 2.1.0. [p112]

D. Kahle and H. Wickham. ggmap: Spatial visualization with ggplot2. The R Journal, 5(1):144-161, 2013. URL https://journal.R-project.org/archive/2013-1/kahle-wickham.pdf. [p103]

M. U. Kemp, E. E. van Loon, J. Shamoun-Baranes, and W. Bouten. RNCEP: Global weather and climate data at your fingertips. Methods in Ecology and Evolution, 3(1):65-70, 2011. doi: 10.1111/j.2041210x.2011.00138.x. [p102]

L. Komsta. outliers: Tests for Outliers, 2011. URL https://CRAN. R-project. org/package=outliers. R package version 0.14. [p114]

T. Leeper, S. Chamberlain, P. Mair, K. Ram, and C. Gandrud. CRAN Task View: Web technologies and services, 2016. URL https: //CRAN. R-project. org/view=WebTechnologies. Version 2016-08-18. [p102] 
O. Mersmann. microbenchmark: Accurate Timing Functions, 2015. URL https: //CRAN. R-project. org/ package=microbenchmark. R package version 1.4-2.1. [p109]

D. Nüst, C. Stasch, and E. J. Pebesma. Connecting R to the sensor web. Lecture Notes in Geoinformation and Cartography, pages 227-246. Springer, 2011. [p114]

Open Geospatial Consortium. OpenGIS geography markup language (GML) encoding standard. Technical report, 2013. URL http://www. opengeospatial.org/standards/gml. [p103]

E. Pebesma. spacetime: Spatio-temporal data in R. Journal of Statistical Software, 51(7):1-30, 2012. doi: 10.18637/jss.v051.i07. [p114]

E. J. Pebesma and R. S. Bivand. Classes and methods for spatial data in R. R News, 5(2):9-13, 2005. URL https://CRAN.R-project.org/doc/Rnews/Rnews_2005-2.pdf. [p103]

J. A. Ryan and J. M. Ulrich. xts: eXtensible Time Series, 2014. URL https://CRAN. R-project.org/ package=xts. R package version 0.9-7. [p103]

K. R. Ryberg and A. V. Vecchia. waterData: An R Package for Retrieval, Analysis, and Anomaly Calculation of Daily Hydrologic Time Series Data, 2014. URL https: //CRAN. R-project. org/package=waterData. R package version 1.0.4. [p102]

K. Slowikowski. ggrepel: Repulsive Text and Label Geoms for "ggplot2", 2016. URL https://CRAN. Rproject. org/package=ggrepel. R package version 0.5. [p103]

A. G. Stephenson. evd: Extreme value distributions. R News, 2(2):31-32, 2002. URL https: //CRAN. Rproject.org/doc/Rnews/Rnews_2002-2.pdf. [p114]

C. Vitolo. rnrfa: UK National River Flow Archive Data from R, 2016. URL https: //CRAN. R-project. org/ package=rnrfa. R package version 1.3.0. [p102]

C. Vitolo, Y. Elkhatib, D. Reusser, C. J. A. Macleod, and W. Buytaert. Web technologies for environmental big data. Environmental Modelling \& Software, 63:185-198, 2015. doi: 10.1016/j.envsoft.2014.10.007. [p102]

H. Wickham. ggplot2: Elegant Graphics for Data Analysis. Springer-Verlag, 2009. URL http://ggplot2. org. [p103]

H. Wickham. The split-apply-combine strategy for data analysis. Journal of Statistical Software, 40(1): 1-29, 2011. doi: 10.18637/jss.v040.i01. [p103]

H. Wickham. httr: Tools for Working with URLs and HTTP, 2016a. URL https: //CRAN. R-project. org/ package=httr. R package version 1.1.0. [p103]

H. Wickham. stringr: Simple, Consistent Wrappers for Common String Operations, 2016b. URL https: //CRAN.R-project.org/package=stringr. R package version 1.1.0. [p103]

H. Wickham and W. Chang. devtools: Tools to Make Developing R Packages Easier, 2016. URL https: //CRAN.R-project.org/package=devtools. R package version 1.12.0. [p103]

H. Wickham and R. Francois. dplyr: A Grammar of Data Manipulation, 2015. URL https://CRAN. Rproject. org/package=dplyr. R package version 0.4.3. [p102]

H. Wickham and J. Hester. xml2: Parse XML, 2016. URL https://CRAN. R-project. org/package=xml2. $\mathrm{R}$ package version 1.0.0. [p103]

C. O. Wilke. cowplot: Streamlined Plot Theme and Plot Annotations for "ggplot2", 2016. URL https: //CRAN.R-project. org/package=cowplot. R package version 0.6.2. [p103]

Y. Xie. Implementing Reproducible Computational Research, chapter knitr: A Comprehensive Tool for Reproducible Research in R. Chapman and Hall/CRC, 2014. [p103]

Y. Xie. DT: A Wrapper of the JavaScript Library "DataTables", 2015a. URL https: //CRAN. R-project.org/ package=DT. R package version 0.1. [p102]

Y. Xie. Dynamic Documents with $R$ and knitr. Chapman and Hall/CRC, 2nd edition, 2015b. ISBN 978-1498716963. [p103]

Y. Xie. knitr: A General-Purpose Package for Dynamic Report Generation in R, 2016. URL https: //CRAN. Rproject. org/package=knitr. R package version 1.14. [p103] 
Claudia Vitolo

Forecast Department

European Centre for Medium-range Weather Forecast

Reading

United Kingdom

claudia.vitolo@ecmwf.int

Matthew Fry

Centre for Ecology and Hydrology

Wallingford

United Kingdom

mfry@ceh.ac.uk

Wouter Buytaert

Department of Civil and Environmental Engineering

Imperial College London

United Kingdom

w. buytaert@imperial.ac.uk 